\title{
A Knowledge Based Document Preparation for Supporting Systems Using Artificial Intelligence
}

\author{
Dawodu, A. \\ Department of Computer Science \\ D.S. Adegbenro ICT Polytechnic \\ Itori, Ewekoro, Nigeria \\ E-mail: babstoncity@gmail.com \\ Phone: +2348132327110
}

\begin{abstract}
A knowledge based way of preparing documents tools in an organization within an activities such as document preparation that are supported by a knowledge based system. Software called REGENT (Report Generation Tool) works in an environment that generates documents from the reusable document pieces during planning, execution and monitoring the document preparation process in a firm or organizational environment. The documents are built from stored document pieces by using artificial intelligence methods. A system architecture was developed to enable the document generation process to take place within a widen office automation standard. The report preparation process knowledge is captured in form of representing a knowledge based scheme. An artificial intelligence problem solving strategy was developed to take care of reasoning steps when document pieces were being configured. The REGENT environment is normally working when preparing a recurrent report types such as annual reports preparation.
\end{abstract}

Keywords: Knowledge based approach, artificial intelligence, document preparation, office automation, document analysis, document standardization.

Proceedings Reference Format

Dawodu, A. \& (2021): A Knowledge Based Document Preparation for Supporting Systems Using Artificial Intelligence.. Proceedings of the 27 th iSTEAMS Multidisciplinary Innovations \& Technology Transfer (MINTT) Conference. Academic City University College, Accra, Ghana. June, 2021. Pp 195-200 www.isteams.net/ghana2021. DOI - https://doi.org/ 10.22624/AIMS/iSTEAMS-2021/V27P16

\section{BACKGROUND TO THE STUDY}

Artificial Intelligence (Al) is an area of computer science that emphasizes the creation of intelligent machines that works and reacts like humans. Some of the activities with the introduction of artificial intelligence in a computer are speech recognition, planning, learning and problem solving. However, it is evident that there is need to explore the possibilities of incorporating knowledge based advantage in our offices today. The reason is to support the recurring document preparation activities in a cooperative and system settings, such as facilitating the update of yearly reports. The design of such an office automation tool not required to take into account the document processing tools and also how the tool can be integrated with the existing information system software and hardware as well as the existing office procedures that exist in an organization. These include an interface with database systems so as to facilitate the storage and retrieval of data in addition to document pieces. 


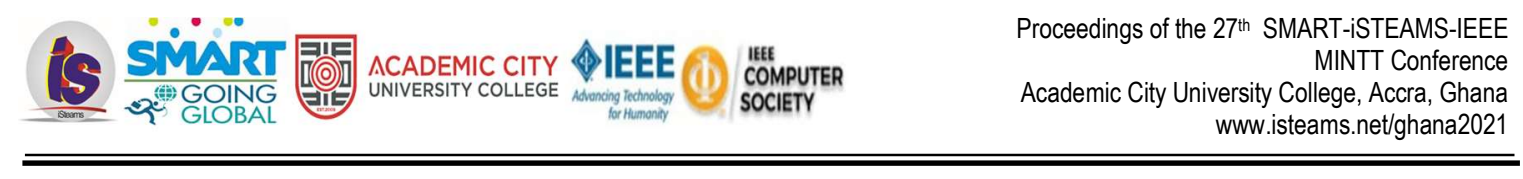

This research work talked about the requirements of such knowledge based system environment which includes integration of various technologies associated with the document preparation application, improving the office work and then the office procedures including the reuse of past paper captured in a document pieces and also supporting the communication among system components.In a situation where recurring nature of reports were observed, a knowledge based approach is used for automating the preparation of such documents. More so, the reports are highly structured and their structure follows predictable patterns. However, the reports can be classified based on the common features, allowing different or same subject matter to be expressed in many versions in application areas. In fact, the contents of the report in addition with report structure also form the report generation process. The approach here considers the document preparation process as an artificial intelligence design task. More importantly, a conceptual model was developed to identify the report components, using appropriate artificial intelligence in a problem solving methodology which was proposed to guide the process and composing reports from report pieces. There are rules and regulations that are enforced through application domains, which are to be connected to during the process of document generations. Also rules and procedures govern an organization in a knowledge based document preparations.

\subsection{Research Aim and Objectives}

The aim of this research work is to develop the conceptual model including its relationship to the presentation model and also to capture the content information within an organizational setting.

\subsection{Objectives}

The main focus of this work is to come up with a better understanding of the specific application relations of the objects in addition to the standard norm descriptions. Also, to focus on applications and standards that are widely used and have been widely deployed within the knowledge based, stored in general and terminal topic definition, being utilized when deciding on the task plan and then carried it out at the time the report is joined together from document pieces.

\section{LITERATURE REVIEW}

\subsection{Concept of Document Standardization}

The two standardized and internationally accepted document norms, the standard generalized markup language (SGML) and the office (OPEN) Document architecture (ODA) and interchange format (ODIF) employs the logical structure model. The norm defines a document class which guides the construction of syntactically correct reports of this document class. ODA and SGML norms do not employ semantics knowledge, except the ODA layout objects. The document norms only include structural rules; as such they neglect the organization they are modeling including the office procedures. These are the two models, the conceptual and presentation. The presentation model is corresponds to the document appearance, thus, it encompasses the logical and layout model. However, the prepared approach to document modeling does not depend on specific presentation model; it can be conceptual model expresses the information requirement of reports preparation process within an office setting in a high level format. All reports belong to a class document while the report structures are represented as frames. The process which recursively combines report components is called the document structuring process. More so, the conceptual model relates report components to organizational procedures. The conceptual model views a document as composed of report pieces called topics. Topics correspond to items listed in the report outline. 
Note that topics are not the same as logical objects because logical objects are part of the presentation model and can take different presentation forms such as text or image. However, the objects are activated by rules during the document generation and structuring process. These rules have two main roles;

i. $\quad$ Function as production rules when generating a new version of the report.

ii. Used to maintain the integrity of the report when modifying report structures.

Moreover, the information that is important to authors is viewed in four ways;

i. $\quad$ Content - the subject matter of the report

ii. Structure - refers to the organization of the material

iii. $\quad$ Format - pertains to the appearance of the report

iv. Meta information - the auxiliary information that is not generally part of what the reader of the report saw, but it is essential to the development of the report structure.

\subsection{Report Model: Strategy in solving problems.}

A knowledge based tool can offer support while designing report context and structure and there is need to select a problem solving method and problem solving process, specifically as a design task, where a report is designed from report pieces. The design task involved in deciding on what information to include as well as organizing this information in a structured document for which would ensure effective communication of report content to readers. These system offer predefined templates that can be modified by users. They do not offer any guidance as how to adapt these templates when modeling new requirements.

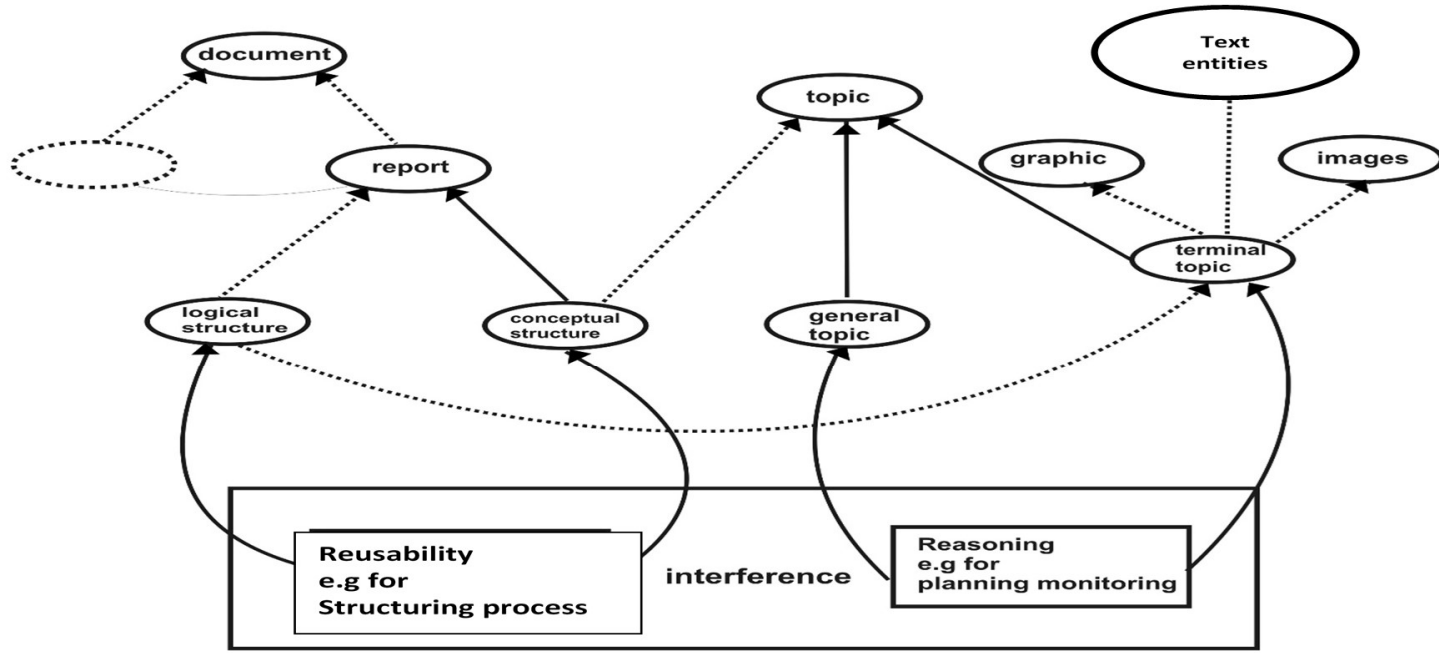

Fig. 1. The report model: ontological structure

- Value class

In an ideal situation, a report generation tool should provide help in building the report structure corresponding to user requirements in a specific situation by supporting the adaptation of predefined templates. These tools can be enhanced to offer support pertaining to adaptation and modification of predefined templates only if they are equipped with a semantic knowledge that can guide the process. 


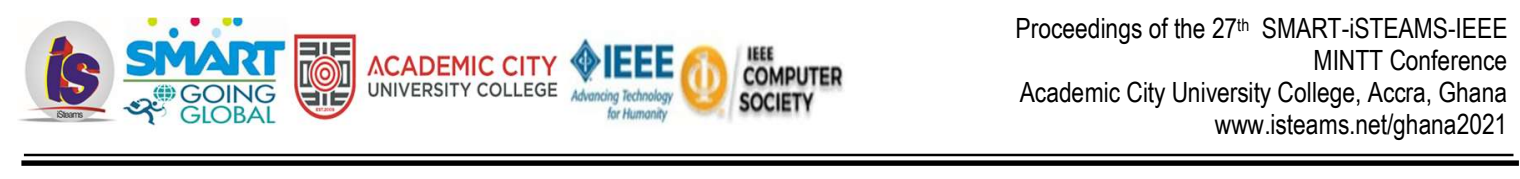

This level of sophistication can be achieved if the report preparation tool is equipped with semantic report structuring capabilities captured in a suitable knowledge representation scheme along with the adaption of a suitable problem solving strategy. However, the letter specifies way and manages the system carried out the reasoning by assuring the role of a user, when adapting the role of a user when adapting the stored document pieces to new organization. This requires the tool to be useful, capable of constructing a report which identify, integrate, instantly support and sequencing the report.Finally, the combination of classification and constructive approach suitable in complex application domains where classification categories can be identified and the interactions can be specified. The approach is applied to such domains as medical diagnostics and intelligent model building decision support systems and others. Figure 1 shows the report model of ontological structure of a document model.

\section{METHODOLOGY}

\subsection{System Architecture}

The system architecture of REGENT is in relation to the situation of office automation support. This architecture is composed of three components;

1. The flexible task management (FTM) component which carries out the exchange of data, procedure activation and message interpretation among the system components.

2. The knowledge management system KMS captures the static knowledge needed during the process of document structuring and construction.

3. The flexible Task Processing (FTP) components utilizes the static knowledge stored in KMS and configured a plan to achieve the goal. This component captures the dynamic aspects of the document construction process, which involves the planning, execution and monitoring of the task.

The report model describes the interaction between various knowledge sources which include database, users and network.

\subsection{The Knowledge Based Component}

The RBC is made up of two parts namely the knowledge management system which is a static system component that stores knowledge to document pieces and related rules whereas the FTP component manages the dynamic part of the report configuration process. However, the KMS captures and represent the knowledge pertaining to the descriptional and procedural parts of the task environment. This information is necessary in order to adequately model the application domain knowledge in an organizational setting.

Moreover, the FTP component manages the dynamics of report configuration and composed of three types that are responsible for document structuring, task planning and task execution and monitoring. The sub components are;

i. Document structuring component that provides the specification language that allows the user to describe the document that is of interest by using the knowledge about report types and features, the system takes over the document structure and presents to the user the likely document structure including the relevant topics and their organizations.

ii. Task planning component carries out the tasks involved in two steps, firstly the task selection component that interprets the findings of the document structuring step and identifies the required activities. Secondly, the configuration component schedules the identified activities by taking into account the dependencies among the activities, then forming a plan fragment.

iii. Task Execution and Monitoring Components controls the execution tasks. The plan execution requires all the activities of all the involved components. The execution control components receive as input 
the partially initiated plan. At this point, supplementary information that would facilitate monitoring and coordination is also considered here.

\section{a. Database Component}

Database management system (DBMS) plays an important role in supporting the office application. It has the potential to facilitate the management structured document pieces and also the management of multimedia objects and hypertext documents. The main focus here is to achieve an integrated interface using object oriented techniques. Also, within the context of document processing with database component that supports the request produced by the knowledge base component when joined together with the model structure.

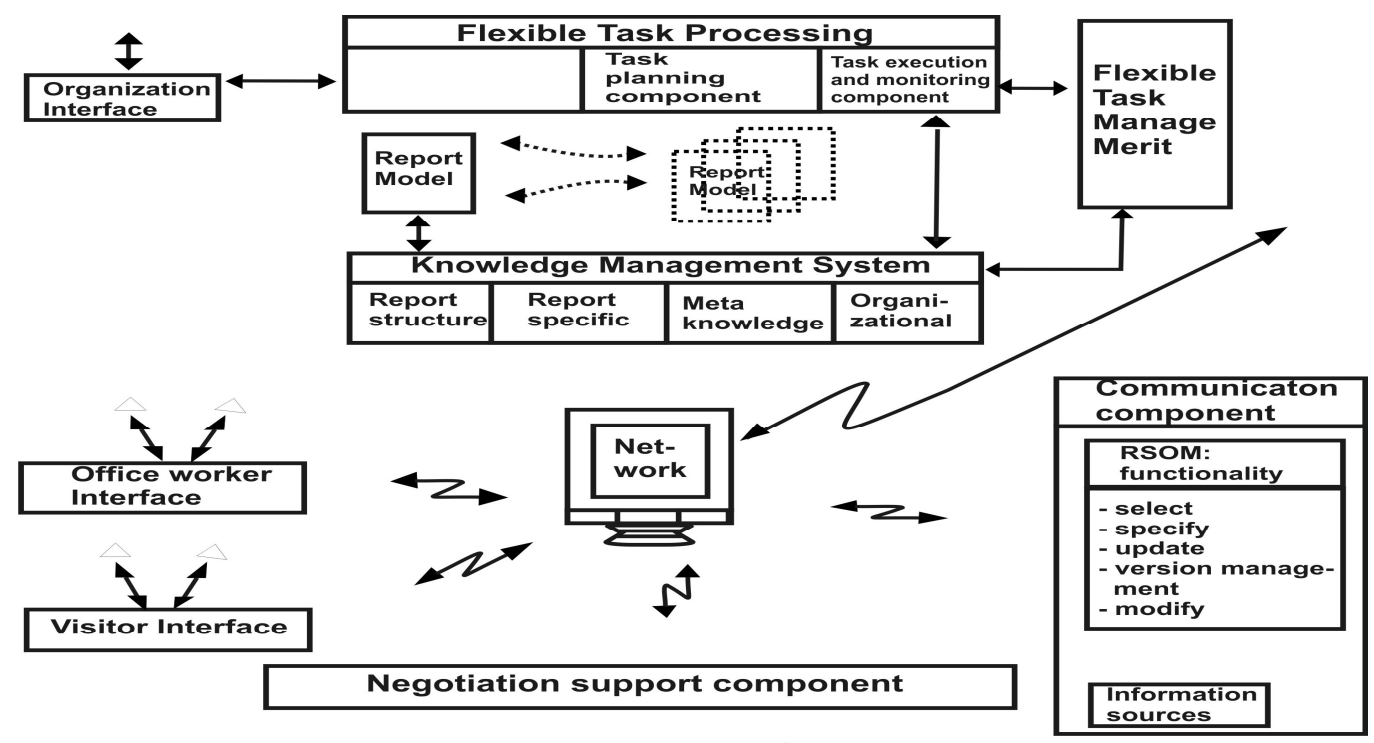

Fig 2; Database Design Components

In addition, the DBMS supports the requests invoked by the users and arrange programs together with data maintenance tasks with communication components that support the internal communication among the system components, which needs the required specific, object oriented model (RSOM), user interface model and the interconnection model. The interconnection model supports various interconnection types including black board and message passing. The blackboard architecture is used to automatically update the knowledge base. Figure 2 shows the system architecture:

\section{iv. DISCUSSION OF FINDINGS}

From the research we found out that emphasis must be placed on the following:

(a) Organizational knowledge about departments and positions, projects and events

(b) Knowledge about individual members of staff

(c) Knowledge about non-human resources such as office technologies as well as documents, standard forms and databases.

(d) Knowledge about office procedures and tasks.

(e) Knowledge about goals and perspectives of the enterprise. 


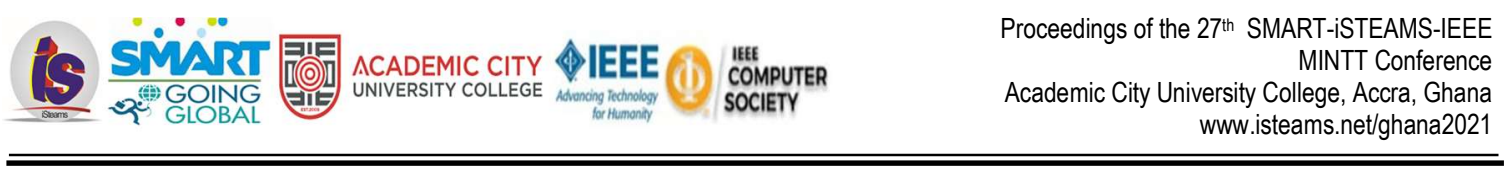

\section{CONCLUDING REMARKS}

Pursuant to the findings from this knowledge based research that the knowledge gathered during the design and little implementation of knowledge based that support the tool for documents was developed together with the recommendation of a two-phased artificial intelligence solving problem strategy. The office automation tool, a system that aims to facilitate the communication among the office work in augmenting the knowledge representation with the existing office procedures. The document model was used to capture the semantic knowledge in document pieces and then used to guide the report composition process. More so, the approach supports in building organizational memory in which the past and the current projects are well represented. A tool was developed, an object - oriented DBMS and network software that promotes the decomposing document which builds several document types and composing a final document pieces.

\section{SUGGESTIONS FOR FURTHER WORKS}

For the purpose of future studies, there is need to study the nature and relationships between business activities with the aim to design and develop a system that can easily support the integration of all office activities, using Neural and Expert Systems.

\section{BIBLIOGRAPHY}

1. Adeyinka, O., "Internet Attack Methods and Internet Security Technology". Modeling and Simulation. 2008 AICMS 08. Second Asia International Conference on vol., no., pp. 77-82, 13-15 May 2008.

2. Alshahwan, N., and Harman, M. (2011). Automated web Application testing using search based software Engineering. 26th IEEE/ACM International Conference on Automate Software Engineering Lawrence, Kansas, USA.

3. Chawla, S. (2011). Design and Development of Business Intelligence portal in Cloud Computing Environment, Thapar University Patiala - 147004

2. Gagliardi, $F$ (2011). Instance-based classifiers applied to Medical databases: Diagnosis and knowledge extraction, Artificial Intelligence in Medicine.,123-139.doi:10.1016/j.artmed

3. Hussian Sandhya Sharma, and Vinita Sharma (2013). "Automated Intelligent Traffic Control System using Sensors". International Journal of Soft Computing and Engineering (IJSCE). 3(3), pp.2231-2307.

4. Khilari, P., Bhope, V. P. (2015). A review on speech to text conversion methods. International Journal of Advanced Research in Computer Engineering \& Technology (IJARET), 4(7)

5. Lin Dong and Wushan Chen (2010). "Real Time Traffic Signal for Urban Road Multi-Intersection" Journal of Intelligent Information Management, pp. 2 483-486. (http://www.scrip.org/journal /iim). May 52012

6. Mircea, M., Ghilic-Micu, B \& Stoica, M. (2011). Combining business intelligence with cloud computing to deliver agility in actual economic. Journal of Economics Computation and Economic Cybernetics Studies, 45(1), 39-54

7. Muthuseilvi, G., \& Saravanan, B. (2014). Real Time Speech Recognition Based Building Automation System. ARPN Journal of Engineering and Applied Sciences, 9(12), 2831-2839

8. Negah, S. (2004). Business Intelligence System. The Communications of the Association for Information Systems, 13(1). 54

9. Orbit-Computer Solutions. 2012. Threats to physical and network infrastructure. Retrieved:

10. Oyelade, O. N., Junaidu, S. B., \& Obiniyi, A. A. (2014). Sematic framework for E- Commerce based on web Ontology Language vol 11 issue 3 No 2. International Journal of Computer Science issues, 1694-0784.

11. Russel, S., Norvig, P.; (1995). Artificial Intelligence. A modern approach. Prentice Hall.

12. Shell Protocol, Asian Journal of Computer and Information System (ISSN: 2321 - 5658) Volume 01 - Issue 04

13. Upadhyay, M. S. K. \& Chavda, M. V. N (2014). 'INTELLIGENT SYSTEM BASED ON SPEECH RECOGNITION WITH CAPABILITY OF SELFLEARNING'. International journal for technological Research in Engineering, 1(9) 